\title{
DIFERENCIAS EN ANSIEDAD ESCOLAR EN FUNCIÓN DEL SEXO Y DEL CURSO ACADÉMICO EN EDUCACIÓN PRIMARIA
}

\author{
Må Isabel Gómez-Núñez \\ Universidad Católica San Antonio de Murcia \\ M. P. Aparicio-Flores \\ Universidad de Alicante \\ María Vicent \\ Universidad de Alicante \\ David Aparisi-Sierra \\ Universidad de Alicante \\ Aitana Fernández-Sogorb \\ Universidad de Alicante \\ Cándido, J. Inglés \\ Universidad de Alicante \\ https://doi.org/10.17060/ijodaep.2017.n1.v3.974
}

Fecha de Recepción: 9 Marzo 2017

Fecha de Admisión: 1 Abril 2017

\section{RESUMEN}

La ansiedad escolar se define como un conjunto de respuestas cognitivas, psicofisiológicas y motoras que un individuo emite ante situaciones evaluadas como amenazantes, peligrosas 0 ambiguas, aunque objetivamente no lo sean. Las importantes consecuencias negativas a corto y largo plazo, que puede provocar la manifestación continuada de esta emoción, aumentan la importancia de su estudio. Así, el propósito principal de esta investigación fue determinar las diferencias en ansiedad escolar según el sexo y el curso académico en una muestra de estudiantes de Educación Primaria. Para ello, se reclutó una muestra de 758 estudiantes de $3^{\circ}, 4^{\circ}, 5^{\circ}$ y $6^{\circ}$ de Educación Primaria con edades comprendidas entre los 8 y los 11 años $(M=9.68, D T=1.23)$. Los participantes completaron el nuevo Inventario de Ansiedad Escolar para Educación Primaria. Los resultados de los análisis de varianza (ANOVA) intersujetos indicaban que las niñas mostraban niveles significativamente superiores de ansiedad escolar en comparación con los niños. Estos niveles de ansiedad escolar aumentaban desde tercero hasta sexto curso, obteniendo los niveles más altos en el último curso de la etapa. Los análisis post-hoc (Prueba de Scheffee) mostraron diferencias estadísticamente significativas entre tercero y quinto curso, así como entre tercero y sexto curso, siendo siempre superiores las medias de los cursos más altos. Estos hallazgos muestran la importan- 
cia de evaluar la ansiedad escolar en la infancia tardía, teniendo en cuenta las diferencias existentes según el sexo y el curso académico.

Palabras clave: Ansiedad escolar, infancia, Educación Primaria, sexo, curso académico, diferencias.

\begin{abstract}
Differences in school anxiety according to sex and academic year in Primary Education

School anxiety is defined as combination of cognitive, psychophysiological and behavioral responses that a person shows in situations assessed as threatening, dangerous or ambiguous, although they were not objectively (Garcia-Fernández, Inglés, Martínez-Monteagudo, \& Redondo, 2008). The important short and long-term negative consequences, which can be caused by the continued manifestation of this emotion, increase the importance of its study. Thus, the main purpose of this research was to determine the differences in school anxiety according to sex and the academic year in a sample of Primary Education students. For this purpose, a sample of 758 students in $3^{\circ}, 4^{\circ}, 5^{\circ}$ and $6^{\circ}$ grades of Primary Education with ages ranging from 8 to $11(M=9.68, S D=1.23)$ was taken on. Participants completed the new School Anxiety Inventory for Primary Education (Gómez-Núñez, García-Fernández, \& Inglés, 2015). The results of the analysis of variance intersubjectives (ANOVA) indicated that girls showed significantly higher levels of school anxiety compared to boys. These levels of school anxiety increased from third to sixth grade, with the highest levels in the last year of the stage. The post-hoc analyzes (Scheffee test) showed statistically significant differences between third and fifth year, as well as between third and sixth year, with the highest means in last years. These findings show the importance of assessing school anxiety in late childhood, taking into account the differences that exist according to sex and the academic year.
\end{abstract}

Keywords: School Anxiety, childhood, Primary Education, sex, academic year, differences.

La ansiedad escolar se define como un conjunto de respuestas cognitivas, psicofisiológicas y motoras que un individuo emite ante situaciones evaluadas como amenazantes, peligrosas y/o ambiguas, aunque objetivamente no lo sean (García-Fernández, Inglés, Martínez-Monteagudo, \& Redondo, 2008). Psicólogos y educadores remarcan la necesidad de estudiar la ansiedad escolar en la infancia debido, principalmente, a las importantes consecuencias negativas, educativas y clínicas, tanto a corto, como a largo plazo, que puede tener la manifestación prolongada e intensa de esta emoción (Miller \& Jome, 2008; Miller \& Jome, 2010; Miller \& LaRae, 2010). Así, las diversas investigaciones demuestran que los niños y adolescentes que manifiestan ansiedad escolar podrían presentar un rendimiento académico más bajo (Freudenthaler, Spinath, \& Neubauer, 2008; Kaplan, Liu, \& Kaplan, 2005; Ratanova \& Likhacheva, 2009), rechazo escolar asociado a la evitación de experiencias negativas en el colegio (García-Fernández, Inglés, Gonzálvez, Vicent, Delgado, \& GómezNúñez, 2016; Kearney, 2002; Kearney, 2007) y otros trastornos emocionales como la ansiedad por separación o la depresión (Isolan, Salum, Osowski, Amaro, \& Manfro, 2011; Martínez-Monteagudo, García-Fernández, \& Inglés, 2013; Nair et al., 2013; Orgilés, Espada, García-Fernández, \& Méndez, 2009).

Centrándonos en las diferencias existentes en la manifestación de este trastorno, numerosos estudios demuestran que las chicas, independientemente del grupo de edad al que pertenezcan, manifiestan mayores niveles de ansiedad escolar que los chicos (Brumariu \& Kerns, 2010; Freudenthaler et al., 2008; Fujita et al., 2009; García-Fernández, Espada, Orgilés, \& Méndez, 2010; García-Fernández, Inglés, Lagos-San Martín, Gonzálvez, Vicent, \& Gómez-Núñez, 2016; Isolan et al., 
2011; Leikanger, Ingul, \& Larsson, 2012; Leikanger \& Larsson, 2012; Lowe, Grumbein, \& Raad, 2011; Orgilés, Espada, Méndez, \& García-Fernández, 2008). Los autores barajan tres posibles hipótesis que explicarían dichas diferencias: La primera de ellas es la hipótesis biológica, que establece que las mujeres y los hombres son diferentes debido a sus propias características genéticas. La segunda hipótesis es de carácter sociocultural, estableciendo que las diferencias entre chicos y chicas en la manifestación de ansiedad son el resultado de los distintos patrones de socialización desarrollados con ambos sexos. Por último, se menciona la hipótesis psicológica, que alude a variables psicológicas (mayores índices de depresión o de trastornos psicológicos) como principales causas de las diferencias entre ambos sexos.

Siguiendo con el estudio de las diferencias existentes en ansiedad escolar, no podemos pasar por alto las diferencias según la edad de los sujetos. En este sentido, los distintos trabajos destacan que, a pesar de que un niño pueda manifestar esta emoción a cualquier edad, lo cierto es que existen diversos momentos en los que podría presentarse de forma más acentuada: en torno a los 5 y 6 años, por el cambio a la etapa de Educación Primaria, lo que puede implicar un cambio de colegio y, en algunos casos, el inicio de la escolaridad (Fremont, 2003; Heyne, King, Tongue, \& Cooper, 2001), entre los 10 y los 12 años, coincidiendo con el paso de la etapa de Educación Primaria a la etapa de Educación Secundaria (Fremont, 2003; García-Fernández et al., 2010; Isolan et al., 2011; Leikanger et al., 2012; Nelemans et al., 2014; Orgilés et al., 2008) 0 entre los 15 y los 17 años, coincidiendo con el final de la adolescencia (García-Fernández, Martínez-Monteagudo, \& Inglés, 2011)

La diversidad de datos existente, así como la necesidad de establecer las diferencias según sexo y edad en ansiedad escolar en la infancia tardía, como periodo determinante en el desarrollo de los niños y niñas, hace patente la importancia de este estudio.

Por ello, el objetivo de esta investigación fue analizar las diferencias en ansiedad escolar según sexo y curso académico en niños de la etapa de Educación Primaria. Según la literatura científica consultada, se espera que: (a) las niñas puntúen significativamente más alto en ansiedad escolar en comparación con los niños; (b) las puntuaciones más altas de ansiedad escolar se hallen en sexto curso, coincidiendo con el último curso de la etapa de Educación Primaria.

\section{MÉTODO}

\section{Participantes}

Se realizó un muestreo aleatorio por conglomerados en las provincias de Albacete, Alicante y Murcia. Las unidades primarias correspondieron a las zonas geográficas de las provincias, las secundarias a los colegios y las unidades terciarias a las aulas en las que se aplicó el instrumento. En un principio, se reclutaron un total de 789 alumnos de tercero, cuarto, quinto y sexto de Educación Primaria, de los cuales 19 (2.40\%) fueron excluidos por no obtener el consentimiento informado de sus padres y $16(2.02 \%)$ por errores u omisiones en sus respuestas. Para ello, se reclutó una muestra de 758 estudiantes de Educación Primaria con edades comprendidas entre los 8 y los 11 años $(M=9.68, D E=1.23)$.

La prueba Chi-cuadrado de homogeneidad de la distribución de frecuencias reveló la ausencia de diferencias estadísticamente significativas entre los ocho grupos de Sexo $\times$ Curso, $\chi^{2}=.83, p=$ .75 (véase Tabla 1). 


\section{DIFERENCIAS EN ANSIEDAD ESCOLAR EN FUNCIÓN DEL SEXO Y DEL CURSO ACADÉMICO EN EDUCACIÓN PRIMARIA}

Tabla 1

Distribución de frecuencias (y porcentajes) Sexo x Curso

\begin{tabular}{|c|c|c|c|c|c|}
\hline & \multicolumn{4}{|c|}{ Curso } & \\
\hline Sexo & $3^{\circ}$ & $4^{\circ}$ & $5^{\circ}$ & $6^{\circ}$ & Total \\
\hline Chico & $75(9.9 \%)$ & $85(11.2 \%)$ & $95(12.5 \%)$ & $115(15.2 \%)$ & $370(48.8 \%)$ \\
\hline Chica & $78(10.3 \%)$ & $89(11.7 \%)$ & $101(13.3 \%)$ & $120(15.9 \%)$ & $388(51.2 \%)$ \\
\hline Total & $153(20.2 \%)$ & $174(22.9 \%)$ & $196(25.8 \%)$ & $235(31.1 \%)$ & $754(100 \%)$ \\
\hline
\end{tabular}

\section{Instrumentos}

El Inventario de Ansiedad Escolar para Educación Primaria (IAEP; Gómez-Núñez et al., 2015) es un instrumento que permite evaluar la ansiedad escolar en niños con edades comprendidas entre los 8 y los 11 años. Este instrumento contiene 19 ítems referidos a situaciones que frecuentemente pueden provocar ansiedad en el colegio y 14 ítems que muestran diferentes respuestas de ansiedad escolar. Los ítems referidos a las situaciones escolares ansiosas se agrupan en cuatro factores: Ansiedad ante el Castigo escolar, Ansiedad ante la Victimización, Ansiedad ante la Evaluación Social y Ansiedad ante la Evaluación Escolar. Por otro lado, los tres sistemas de respuesta muestran reacciones cognitivas, reacciones psicofisiológicas y reacciones motoras.

Gómez-Núñez et al. (2015) analizaron la validez de la escala a través de análisis factoriales exploratorios (AFE) y análisis factoriales confirmatorios (AFC), obteniendo índices de consistencia interna (Alpha de Cronbach) que resultaron aceptables tanto para el total de la escala $(\alpha=.92)$, como para los cuatro factores situacionales $(\alpha=.85-.90)$ y para las tres escalas de respuesta $(\alpha=.80-.84)$.

\section{Procedimiento}

Una vez establecidos los propósitos e hipótesis de trabajo, se llevó a cabo una entrevista con los directores, jefes de estudios y profesorado de los centros educativos seleccionados. Posteriormente, se envió una carta informativa a los padres de los estudiantes para explicarles el estudio y solicitar su consentimiento informado por escrito. Tras la conclusión del proceso de selección de la muestra de participantes y la obtención del consentimiento informado, se procedió a la administración de la prueba. El instrumento fue administrado de manera voluntaria y colectiva en el aula a todos los alumnos y alumnas incluidos en la muestra que finalmente formaron parte de la investigación. Cada una de las aulas estuvo formada por una media de 21 alumnos.

De este modo, se procedió a la entrega de los ejemplares con las instrucciones pertinentes. En primer lugar, se indicó que cumplimentasen los datos de identificación (número de identificación, sexo, edad, curso y centro) y se leyeron en voz alta las instrucciones, resaltando la importancia de no dejar ninguna pregunta sin contestar. La administración de la prueba se llevó a cabo en una sesión de cincuenta minutos.

\section{Análisis de datos}

Para establecer las diferencias según el sexo y curso académico se realizaron análisis de varianza (ANOVA) intersujetos $2 \times 4$ (sexo $x$ curso), así como análisis post-hoc a través de la prueba de Scheffee. Debido a la amplitud de la muestra del estudio, el estadístico $F$ puede detectar errónea- 
mente diferencias estadísticamente significativas. Por esta razón se incluyó el índice $d$ (diferencia media tipificada) propuesto por Cohen (1988), que permite valorar la magnitud o el tamaño del efecto de las diferencias halladas. Los criterios establecidos para su valoración son los siguientes: $.20 \leq d \leq .49$ indicaría una magnitud baja de las diferencias; $.50 \leq d \leq .79$ señalaría una magnitud moderada de las diferencias; $d \geq .80$ indicaría una magnitud alta de las diferencias. Todos los análisis fueron llevados a cabo con el programa SPSS 22.

\section{RESULTADOS}

Tal y como se puede observar en la Tabla 2, las chicas puntuaron significativamente más alto en ansiedad escolar que los chicos $(F=29.86, p<.001)$. Posteriormente, se obtuvo el tamaño del efecto (d) que fue igual a .38. Siguiendo el criterio de Cohen (1988), la magnitud de las diferencias según sexo fue baja $(.20 \leq d \leq .49)$.

Tabla 2

Diferencias según el sexo en ansiedad escolar

\begin{tabular}{|c|c|c|c|c|c|c|c|c|c|}
\hline \multirow[t]{2}{*}{ IAEP } & \multicolumn{2}{|c|}{ Chicos } & \multicolumn{2}{|c|}{ Chicas } & \multicolumn{2}{|c|}{ Total } & \multicolumn{3}{|c|}{$\begin{array}{c}\text { Significación } \\
\text { estadística }\end{array}$} \\
\hline & $M$ & $D E$ & $M$ & $D E$ & $M$ & $D E$ & $F$ & $p$ & $d$ \\
\hline IAEP & & & & & & & & & \\
\hline total & 54.89 & 34.68 & 69.00 & 38.43 & 62.18 & 37.32 & 29.86 & $<.001$ & .38 \\
\hline
\end{tabular}

IAEPtotal $=$ Puntuación total del cuestionario.

De la misma forma, la ansiedad escolar también varió significativamente en función del curso académico, atendiendo a la puntuación total del IAEP. Tal y como muestra la Tabla 3, podemos observar que los niveles más altos de ansiedad escolar se hallaron en sexto curso ( $M=66.37, D E$ $=34.02)$, mientras que los niveles más bajos se encontraron en tercer curso $(M=49.25, D E=$ 22.36). Los análisis post-hoc revelaron diferencias estadísticamente significativas entre $3^{\circ}$ y $5^{\circ}(p<$ $.001)$ y entre $3^{0}$ y $6^{0}$ curso $(p<.001)$, siendo estas diferencias de magnitud baja y moderada respectivamente ( $d=.40$ en el primer caso; $d=.52$ en el segundo caso).

Tabla 3

Diferencias según el curso académico en ansiedad escolar

\begin{tabular}{|c|c|c|c|c|c|c|c|c|c|c|}
\hline \multirow[t]{2}{*}{ IAEP } & \multicolumn{2}{|c|}{$3^{\circ}$ Primaria } & \multicolumn{2}{|c|}{$4^{\circ}$ Primaria } & \multicolumn{2}{|c|}{$5^{\circ}$ Primaria } & \multicolumn{2}{|c|}{$6^{\circ}$ Primaria } & \multicolumn{2}{|c|}{$\begin{array}{c}\text { Significación } \\
\text { estadística }\end{array}$} \\
\hline & $M$ & $D E$ & $M$ & $D E$ & $M$ & $D E$ & $M$ & $D E$ & $F$ & $p$ \\
\hline IAEPtot & 49.25 & 22.36 & 54.10 & 29.78 & 64.05 & 34.51 & 66.37 & 34.02 & 10.01 & $<.001$ \\
\hline
\end{tabular}

IAEPtotal $=$ Puntuación total del inventario. 
Para completar el estudio de las diferencias en los niveles de ansiedad escolar, se examinaron los efectos de interacción Sexo x Curso para el IAEP total (véase Tabla 4, Figura 1). Así, observamos que el efecto de interacción Sexo $x$ Curso fue significativo para la puntuación total del IAEP ( $F$ $=3.15, p<.05)$. Las chicas de sexto presentaban niveles de ansiedad escolar más elevados que el resto de grupos $(M=78.15, D E=37.33)$, mientras que los chicos de tercer curso mostraban los niveles más bajos de esta emoción ( $M=44.46, D E=34.18)$.

Tabla 4

Interacciones Sexo x Curso. Puntuación total IAEP

\begin{tabular}{|c|c|c|c|c|c|c|c|c|c|c|c|}
\hline \multirow[t]{2}{*}{ IAEP } & & \multicolumn{2}{|c|}{$3^{o}$ Primaria } & \multicolumn{2}{|c|}{$4^{\circ}$ Primaria } & \multicolumn{2}{|c|}{$5^{\circ}$ Primaria } & \multicolumn{2}{|c|}{$6^{\circ}$ Primaria } & \multicolumn{2}{|c|}{$\begin{array}{l}\text { Significación } \\
\text { estadística }\end{array}$} \\
\hline & & $M$ & $D E$ & $M$ & $D E$ & $M$ & $D E$ & $M$ & $D E$ & $F$ & $p$ \\
\hline \multirow{2}{*}{ IAEPtot } & Chico & 44.46 & 34.18 & 49.75 & 30.37 & 61.89 & 37.59 & 54.50 & 32.00 & \multirow{2}{*}{3.15} & \multirow{2}{*}{.02} \\
\hline & Chica & 53.97 & 35.10 & 62.19 & 37.79 & 63.79 & 38.46 & 78.15 & 37.33 & & \\
\hline
\end{tabular}

IAEPtotal $=$ Puntuación total del inventario.

Figura 1.

Diferencias en función del sexo y curso académico: Interacciones

Sexo $\times$ Curso en base a la puntuación total del IAEP.

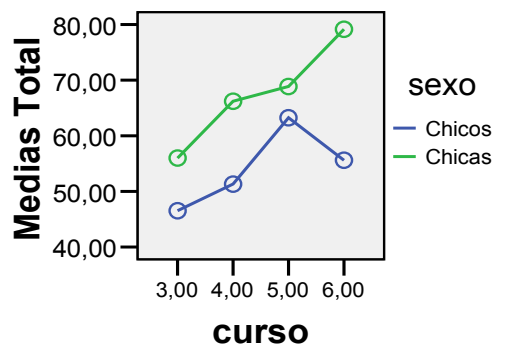

\section{DISCUSIÓN}

La ansiedad escolar se define como uno de los trastornos más importantes a tener en cuenta en la infancia (Miller \& Jome, 2008; Miller \& Jome, 2010; Miller \& LaRae, 2010). Las importantes consecuencias negativas para el desarrollo de los niños justifican su estudio (Freudenthaler et al., 2008; Isolan et al., 2011; Kaplan et al., 2005; Kearney, 2002; Kearney, 2007; Martínez-Monteagudo et al., 2013; Nair et al., 2013; Orgilés et al., 2009; Ratanova \& Likhacheva, 2009). Por ello, el objetivo principal de este trabajo fue analizar las diferencias en ansiedad escolar según sexo y curso académico en niños de la etapa de Educación Primaria.

La primera hipótesis quedó confirmada ya que, tal y como se esperaba, las niñas puntuaron significativamente más alto en ansiedad escolar que los niños. Estos datos resultan coherentes con 
otras investigaciones en este ámbito, las cuales explicaban la presencia de dichas diferencias por tres hipótesis diferenciadas: Hipótesis Biológica, Hipótesis Sociocultural e Hipótesis Psicológica (Brumariu \& Kerns, 2010; Freudenthaler et al., 2008; Fujita et al., 2009; García-Fernández et al., 2010; Isolan et al., 2011; Leikanger et al., 2012; Leikanger \& Larsson, 2012; Lowe et al., 2011; Orgilés et al., 2008). Según la hipótesis biológica, las diferencias se deberían a las propias características genéticas distintivas de hombres y mujeres. Para la hipótesis sociocultural, las diferencias entre chicos y chicas serían consecuencia de los distintos patrones de socialización desarrollados. Las chicas podrían encontrarse más libres a la hora de manifestar este tipo de reacciones emocionales, a lo que debe sumarse la sobreprotección que sus cuidadores ejercen sobre ellas, en muchas ocasiones, aspecto que podría llevarlas a exhibir mayores niveles de ansiedad y de miedo ante situaciones amenazantes. Los chicos, por su parte, son animados a manifestar otro tipo de conductas como la valentía o la agresividad, considerando las conductas asociadas a la ansiedad como signos de debilidad. En último lugar, según la hipótesis psicológica, serían las variables de tipo psicológico las que determinarían las diferencias por sexo. El aumento de los índices de depresión en mujeres, un pobre autoconcepto, así como una baja autoestima asociada a los cambios puberales y la presencia de distorsiones cognitivas, podrían influir en el aumento de los niveles de ansiedad ante situaciones escolares. En este estudio, se aboga por una complementariedad de estas tres hipótesis. Esto se debe, principalmente, a la asunción de que uno solo de estos factores no podría explicar por si solo tales diferencias.

La segunda hipótesis también se confirmó, debido a que las puntuaciones más altas en ansiedad escolar fueron las obtenidas por los alumnos del último curso de la etapa de Educación Primaria. Estos hallazgos son apoyados por otras investigaciones que sitúan los 10-12 años como uno de los momentos más importantes en la aparición de dicha reacción emocional (Fremont, 2003; García-Fernández et al., 2010; García-Fernández et al., 2011; Orgilés et al., 2008). Esto se explica por el cambio que experimentan los niños de esas edades: la transición a una nueva etapa educativa. Los alumnos de sexto curso se enfrentan al paso de la etapa de Educación Primaria a la etapa de Educación Secundaria Obligatoria, hecho que supone para ellos un cambio de profesores, de materias educativas, de centro educativo, un aumento de la exigencia académica y, en la mayoría de los casos, un cambio de compañeros y compañeras, aspectos que podrían influir en la manifestación de mayores niveles de ansiedad escolar. Además, los niños y niñas del último curso de la etapa de Educación Primaria se encuentran en una situación de incertidumbre, de búsqueda de su propia identidad personal y en el comienzo del cambio puberal, más acentuado en las chicas, lo que podría incrementar el estrés en el contexto escolar, considerado como uno de los ámbitos más importantes para su desarrollo. En el caso de los chicos, tal y como pudimos ver en el apartado de resultados, se hallaron niveles superiores de ansiedad escolar en quinto curso, descendiendo posteriormente en sexto. No obstante, debemos tener en cuenta que, los hallazgos en los chicos siguen mostrando niveles de ansiedad superiores en el último tramo de esta etapa educativa, en comparación con tercero y cuarto de Educación Primaria, lo cual resulta coherente con otras investigaciones en este mismo campo de estudio (Fremont, 2003; García-Fernández et al., 2010; Isolan et al., 2011; Leikanger et al., 2012; Nelemans et al., 2014; Orgilés et al., 2008).

Por último, con el fin de analizar de manera exhaustiva las posibles diferencias existentes en los niveles de ansiedad escolar entre los distintos grupos muestrales, es importante valorar los efectos de interacción Sexo x Curso. Así, los resultados revelaron la existencia de efectos de interacción Sexo $\times$ Curso significativos para la puntuación total del IAEP. Esto indicaba que, generalmente, las chicas de sexto de Educación Primaria presentaban las puntuaciones más altas en ansiedad escolar, mientras que los chicos de tercero de Educación Primaria, mostraban las puntuaciones más 
bajas. Estos hallazgos podrían ser explicados por las distintas hipótesis y explicaciones dadas por separado a las diferencias por sexo y por curso académico. Las chicas de sexto manifestarían niveles superiores de ansiedad escolar debido tanto a la etapa de transición en la que se encuentran, como a aspectos biológicos, socioculturales y psicológicos. Por su parte, los chicos de tercero de Educación Primaria manifestarían los niveles más bajos de ansiedad escolar debido a la estabilidad educativa en la que se encuentran, al menor nivel de exigencia académica y a sus características biológicas, socioculturales y psicológicas.

A pesar de la importancia de los resultados obtenidos y de la validez del instrumento empleado, estos deben valorarse con una serie de limitaciones a tener en cuenta en futuras investigaciones en este campo. Por un lado, es necesario considerar que no se examinaron las posibles patologías y las características culturales de la muestra de estudio, aspecto que debería ser estimado en otras investigaciones en este ámbito. En segundo lugar, a pesar de la importancia que muchos investigadores confieren a los autoinformes como instrumentos evaluadores de distintas variables psicoeducativas como la ansiedad escolar (García-Fernández et al., 2008; Miller \& Jome, 2008), lo cierto es que el empleo de estas herramientas no es suficiente a la hora de determinar el grado de severidad de un trastorno. Esto es debido a los sesgos derivados de la deseabilidad social, ya que los alumnos pueden minimizar 0 aumentar sus propios síntomas, dependiendo de sus expectativas acerca de lo que pueden pensar el resto de personas significativas para ellos. En este sentido, sería recomendable que, en futuros estudios, se empleen otras técnicas, como las entrevistas o las técnicas de observación, para realizar un diagnóstico más ajustado sobre los niveles de ansiedad escolar del alumnado, aludiendo así a una perspectiva multimétodo.

No obstante, a pesar de las limitaciones mencionadas, los resultados obtenidos ponen de manifiesto la importancia de valorar, especialmente, los niveles de ansiedad escolar en las chicas y en los alumnos del último tramo de la Educación Primaria (quinto y sexto curso). Los datos mostraban niveles superiores de ansiedad escolar en los grupos mencionados, aspecto que apoya la idea de poner en práctica programas para la mejora de los niveles de ansiedad escolar que tengan en cuenta a todo el alumnado, pero implicando, de una manera más exhaustiva, a las chicas y a los alumnos de quinto y sexto curso. Esto podría prevenir que la ansiedad escolar se convirtiera en un trastorno clínico, reduciendo así los riesgos de un peor pronóstico y desarrollo en la adolescencia.

\section{REFERENCIAS BIBLIOGRÁFICAS}

Brumariu, L. E., \& Kerns, K. A. (2010). Mother-Child attachment patterns and different types of anxiety symptoms: Is there specificity of relations? Child Psychiatry \& Human Development, 41(6), 663-674. doi: 10.1007/s10578-010-0195-0.

Cohen, J. (1988). Statistical power analysis for the behavioral sciences. Hillsdale, NJ: Erlbaum.

Fremont, W. P. (2003). School refusal in children and adolescents. American Family Physician, 68(8), 1555-1560.

Freudenthaler, H. H., Spinath, B., \& Neubauer, A. C. (2008). Predicting School Achievement in Boys and Girls. European Journal of Personality, 22(3), 231-245. doi: 10.1002/per.678

Fujita, M., Fijiwara, J., Maki, T., Shibasaki, K., Shigeta, M., \& Nii, J. (2009). Pediatric chronic daily headache associated with school phobia. Pediatrics International, 51(5), 621-625. doi: 10.1111/j.1442-200X.2009.02804.X.

García-Fernández, J. M., Espada, J. P., Orgilés, M., \& Méndez, X. (2010). Psychometric properties of the School Fears Survey Scale for preadolescents (SFSS-II). Psicothema, 22(3), 502-508.

García-Fernández, J. M., Inglés, C. I., Gonzálvez, C., Vicent, M., Delgado, B., \& Gómez-Núñez, M. I. (2016). Revisión bibliométrica del rechazo escolar: perspectivas de investigación y su análisis. 
Educatio Siglo XXI, 34(1), 71-92.

García-Fernández, J. M., Inglés, C. J., Lagos-San Martín, N., Gonzálvez, C., Vicent, M., \& GómezNúñez, M. I. (2016). Diferencias de sexo y edad en rechazo escolar en una muestra de adolescentes chilenos. Estudios Pedagógicos, 42(1), 127-137.

García-Fernández, J. M., Inglés, C. J., Martínez-Monteagudo, M. C., \& Redondo, J. (2008). Evaluación y tratamiento de la ansiedad escolar en la infancia y la adolescencia. Behavioral Psychology/Psicología Conductual, 16(3), 413-437. doi: http://dx.doi.org/10.5093/ed2013a5

García-Fernández, J. M., Martínez-Monteagudo, M. C., \& Inglés, C. J. (2011). Diferencias según sexo y curso en ansiedad escolar: Estudio con una muestra de estudiantes españoles de Educación Secundaria. Ansiedad y Estrés, 17(2-3), 137-148.

Gómez-Núñez, M. I., García-Fernández, J. M., \& Inglés, C. J. (2015). Inventario de Ansiedad Escolar Para Educación Primaria (IAEP). Manual Interno [Inventory of School Anxiety for Primary Education (IAEP). Internal manual]. Alicante: Universidad de Alicante.

Heyne, D., King, N. J., Tonge, B., \& Cooper, H. (2001). School refusal: Epidemiology and management. Pediatric Drugs, 3, 719-732.

Isolan, L., Salum, G. A., Osowski, A. T., Amaro, E., \& Manfro, G. G. (2011). Psychometric properties of the screen for child anxiety related emotional disorders (SCARED) in brazilian children and adolescents. Journal of Anxiety Disorders, 25(5), 741-748. doi: 10.1016/j.janxdis.2011.03.015.

Kaplan, D. S., Liu, R. X., \& Kaplan, H. B. (2005). School related stress in early adolescence and academic performance three years later: the conditional influence of self-expectations. Social Psychology of Education, 8, 3-17. doi:10.1007/s11218-004-3129-5

Kearney, C. A. (2002). Identifying the function of School Refusal Behavior: A revision of the School Refusal Assessment Scale. Journal of psychopathology and Behavioral Assessment, 24(4), 235245. doi:10.1023/A:1020774932043

Kearney, C. A. (2007). Forms and functions of school refusal behavior in youth: an empirical analysis of absenteeism severity. Journal of Child Psychology and Psychiatry, 48(1), 53-61. doi:10.1111/j.1469-7610.2006.01634.x

Leikanger, E., Ingul, J. M., \& Larsson, B. (2012). Sex and age-related anxiety in a community sample of Norwegian Adolescents. Scandinavian Journal of Psychology, 53, 150-157. doi: 10.1111/j.1467-9450.2011.00915.x.

Leikanger, E., \& Larsson, B. (2012). One-year stability, change and incidence in anxiety symptoms among early adolescents in the general population. European Child \& Adolescent Psychiatry, 21 , 493-501. doi: 10.1007/s00787-012-0284-7.

Lowe, P. A., Grumbein, M. J., \& Raad, J. M. (2011). Examination of the psychometric properties of the Test Anxiety Scale for Elementary Students (TAS-E) Scores. Journal of Psychoeducational Assessment, 29(6), 503-514.

Martínez-Monteagudo, M. C., García-Fernández, J. M., \& Inglés, C. J. (2013). Relaciones entre ansiedad escolar, ansiedad rasgo, ansiedad estado y depresión en una muestra de adolescentes españoles. International Journal of Psychology and Psychological Therapy, 13(1), 47-64.

Miller, D. N., \& Jome, L. M. (2008). School Psychologists and the Assessment of childhood internalizing disorders: Perceived knowledge, role preferences and training needs. School Psychology International, 29, 500-510.

Miller, D. N., \& Jome, L. M. (2010). School Psychologists and the secret illness: Perceived knowledge, role preferences and training needs regarding the prevention and treatment for internalizing disorders. School Psychology International, 31(5), 509-520.

Miller, D., \& LaRae, M. (2008). School psychologists and the assessment of childhood internalizing 
disorders: Perceived knowledge, role preferences and training needs. School Psychology International, 29, 500-510.

Nair, M., Russell, P. S. S., Subramaniam, V. S., Nazeema, S., Chembagam, N., Russell, S.,...Charles, H. (2013). ADad 8: School phobia and anxiety disorders among adolescents in a rural community population in india. The Indian Journal of Pediatrics, 80(2), 171-174. doi: 10.1007/s12098013-1208-3

Nelemans, S. A., Hale, W. W., Branje, S. J., Raaijmakers, Q. A., Frijns, T., van Lier, P. A., \& Meeus, W. H. (2014). Heterogeneity in development of adolescent anxiety disorder symptoms in an 8year longitudinal community study. Development and Psychopathology, 26(1), 181-202. doi: 10.1017/S0954579413000503.

Orgilés, M., Espada, J. P., García-Fernández, J. M., \& Méndez, F. X. (2009). Relación entre miedos escolares y síntomas de ansiedad por separación infantil. Revista Mexicana de Psicología, 26(1), 17-25.

Orgilés, M., Espada, J. P., Méndez, X., \& García-Fernández, J. M. (2008). Miedos escolares en hijos de padres divorciados y no divorciados. International Journal of Clinical and Health Psychology, 8(3), 693-703.

Ratanova, T. A., \& Likhacheva, E. V. (2009). Correlation of School Anxiety with cognitive peculiarities of Junior School Children. Psikholgicheskii Zhurnal, 30(3), 39-51. doi: http://dx.doi.org/10.1016/j.sbspro.2015.02.311 